\title{
Alteration in Lignin Biosynthesis Restricts Growth of Fusarium spp. in Brown Midrib Sorghum
}

\author{
Deanna L. Funnell-Harris, Jeffrey F. Pedersen, and Scott E. Sattler
}

First author: Grain, Forage and Bioenergy Research Unit, United States Department of Agriculture-Agricultural Research Service, 314 BioChem Hall, East Campus, and Department of Plant Pathology, University of Nebraska, Lincoln 68583-0737; and second and third authors: Department of Agronomy and Horticulture, University of Nebraska, Lincoln.

Accepted for publication 13 March 2010.

\begin{abstract}
Funnell-Harris, D. L., Pedersen, J. F., and Sattler, S. E. 2010. Alteration in lignin biosynthesis restricts growth of Fusarium spp. in brown midrib sorghum. Phytopathology 100:671-681.

To improve sorghum for bioenergy and forage uses, brown midrib (bmr)6 and -12 near-isogenic genotypes were developed in different sorghum backgrounds. The bmr6 and bmrl2 grain had significantly reduced colonization by members of the Gibberella fujikuroi species complex compared with the wild type, as detected on two semiselective media. Fusarium spp. were identified using sequence analysis of a portion of the translation elongation factor (TEF) 1- $\alpha$ gene. The pathogens Fusarium thapsinum, F. proliferatum, and F. verticillioides, G. fujikuroi members, were commonly recovered. Other frequently isolated Fusarium spp. likely colonize sorghum asymptomatically. The $\chi^{2}$ analyses showed
\end{abstract}

ABSTRACT that the ratios of Fusarium spp. colonizing bmr12 grain were significantly different from the wild type, indicating that bmr12 affects colonization by Fusarium spp. One $F$. incarnatum-F. equiseti species complex (FIESC) genotype, commonly isolated from wild-type and bmr6 grain, was not detected in $b m r 12$ grain. Phylogenetic analysis suggested that this FIESC genotype represents a previously unreported TEF haplotype. When peduncles of wild-type and near-isogenic $b m r$ plants were inoculated with $F$. thapsinum, $F$. verticillioides, or Alternaria alternata, the resulting mean lesion lengths were significantly reduced relative to the wild type in one or both $b m r$ mutants. This indicates that impairing lignin biosynthesis results in reduced colonization by Fusarium spp. and A. alternata.

Additional keywords: caffeic acid $O$-methyltransferase, cinnamyl alcohol dehydrogenase, Fusarium equiseti.
Sorghum (Sorghum bicolor (L.) Moench) is a valuable crop due to its adaptability to drought-stressed conditions (16). Recently, interest in sorghum grain and biomass has been piqued because of their usability as feed stock for bioenergy industries (54). Genetic modification to reduce lignin content in sorghum and other potential bioenergy crops has promise for greater utilization by increasing biomass digestibility (32). However, lignin is important for structural support and water transport within the plant (7). Additionally, lignin is involved in defense against pathogens and insects by providing a physical barrier $(18,62)$, and lignin biosynthesis is induced in response to biotic stresses (26).

Two different mutated genes in the sorghum lignin biosynthesis pathway have allowed the development of forage and grain lines with significantly reduced lignin content (46). These mutations, brown midrib (bmr)6 and bmr12, result in the phenotype of reddish-brown leaf midveins which are associated with reduced lignin content and modified lignin composition $(46,48)$. Bmr6 encodes cinnamyl alcohol dehydrogenase (CAD) $(53,57)$ and Bmr12 encodes caffeic acid $O$-methyltransferase (COMT) (5). CAD converts the aldehyde group of monolignols to an alcohol for incorporation into the lignin polymer, while COMT methylates the 5-hydroxyl group of the hydroxyl coniferyl substrates forming sinapyl groups (24). Both bmr6 and bmrl2 are caused by nonsense mutations that result in the absence of detectable gene products $(5,48,57)$.

Corresponding author: D. L. Funnell-Harris;

E-mail address: Deanna.Funnell-Harris@ars.usda.gov

doi:10.1094/PHYTO-100-7-0671

This article is in the public domain and not copyrightable. It may be freely reprinted with customary crediting of the source. The American Phytopathological Society, 2010.
When bmr6, bmr12, and near-isogenic wild-type lines were grown at two locations in field trials, density of bmr6 grain was reduced (46), which could be caused by infection by grainmolding pathogens (34). However, when some of this same grain was screened, results indicated that $b m r$ genotypes could have significantly fewer colonies of Fusarium spp. per seed (20). Among species identified using morphological characters were Fusarium thapsinum (formerly known as F. moniliforme), $F$. proliferatum, and $F$. subglutinans, members of the species complex Gibberella fujikuroi $(31,44)$. Greenhouse inoculation of peduncles of bmr6 and bmrl2 plants with $F$. thapsinum resulted in mean lesion lengths significantly smaller than those on nearisogenic wild-type plants (20). When considering genetic background, one or both low-lignin lines had significantly smaller mean lesions as a result of inoculation with $F$. thapsinum in four of six genetic backgrounds. Therefore, this research indicated that bmr resulted in reduced colonization by Fusarium spp. but genetic background influenced the interaction with low-lignin plants (20).

Several Fusarium spp. in the G. fujikuroi species complex are potentially pathogenic on sorghum (31). Infections of stalks or roots can reduce yield and grain quality while lodging can make harvest difficult (6). Infections of panicles can result in grain of reduced quality and usability (36). F. thapsinum and other Fusarium spp., including those considered nonpathogenic, are able to colonize sorghum without producing symptoms $(20,21$, 30). Many Fusarium spp. produce mycotoxins $(31,41)$. Accumulation of these metabolites are often exacerbated when grain is stored or stover is ensiled $(1,13)$. Deterioration by grain-molding fungi and contamination by mycotoxins may be a particular problem in cultures in which sorghum is used in foods and beverages as well as for livestock feed, and grain is stored in traditional containers on rural farms $(14,55)$. Long-term exposure to mycotoxins 
can result in life-threatening diseases to animals and possibly to humans (60).

Because grain of $b m r$ genotypes exhibited reduced colonization by Fusarium spp. and peduncles had reduced colonization by $F$. thapsinum, the hypothesis that these genotypes had specific interactions with Fusarium spp. was proposed. Fusarium isolates from grain of field-grown $b m r$ and wild-type plants were identified to species using sequence analysis of the translation elongation factor (TEF) 1- $\alpha$ gene. Five Fusarium genotypes and one Alternaria sp. commonly found in sorghum fields, plants, or debris were inoculated onto peduncles of $b m r$ plants and nearisogenic wild-type plants under greenhouse conditions. Results of this study may elucidate differential colonization of grain and stalks of $b m r$ and near-isogenic wild-type plants.

\section{MATERIALS AND METHODS}

Fusarium and Alternaria spp. colonization of field-grown grain from $\boldsymbol{b m r}$ and wild-type plants. Plants of the wild type, bmr6, and bmr12 in genetic backgrounds BTx623, BTx631, and Redlan and wild-type, bmr6, bmr12, and bmr6, bmr12 double mutant plants in backgrounds Wheatland and RTx430 were grown in two-row plots, $7.6 \mathrm{~m}$ long and spaced $76 \mathrm{~cm}$ apart, at University of Nebraska Field Laboratories in Lincoln and Ithaca, NE in 2003 (49,50). During 2004, bmr6, bmr12, double-mutant, and wild-type near-isogenic lines in Wheatland and RTx430 backgrounds were grown at both locations.

For 2003, nitrogen fertilizer was applied preplant at both locations at $112 \mathrm{~kg} \mathrm{ha}^{-1}$. At the Lincoln location, propachlor (2-chloro$N$-(1-methylethyl)- $N$-phenylacetamide) and atrazine (6-chloro- $n$ ethyl- $N$ '-(1methylethyl)-1,3,5-trazine-2,4-diamine) were applied at 3.36 and $1.1 \mathrm{~kg} \mathrm{ha}^{-1}$, respectively, immediately after planting for weed control. At Ithaca, atrazine was applied at $2.2 \mathrm{~kg} \mathrm{ha}^{-1}$ immediately after planting, followed by an application of quinclorac (3,7-dichloro-8-quinolinecarboxylic acid) and atrazine at 0.37 and $1.1 \mathrm{~kg} \mathrm{ha}^{-1}$, respectively, $\approx 14$ days postemergence. At Ithaca, supplemental irrigation was applied via overhead sprinklers on 24 July $(2.5 \mathrm{~cm})$ and $4(5.0 \mathrm{~cm}), 7(5.0 \mathrm{~cm}), 14(2.5 \mathrm{~cm})$, and $28(2.5 \mathrm{~cm})$ August. In 2004, nitrogen fertilizer was applied prior to planting at both locations at $157 \mathrm{~kg} \mathrm{ha}^{-1}$ and herbicides were applied as in 2003. Supplemental irrigation $(2.5 \mathrm{~cm})$ was applied at Ithaca on 3,12, and 19 August. These field plantings also were yield studies; therefore, no artificial inoculations were conducted and irrigation was applied to avoid water stress. Grain yields, test weights, and fiber and grain content were previously reported (46,50,56). In 2003, lodging scores approached zero. Visual inspection of grain samples from both years showed no obvious signs or symptoms of grain molding.

For each plot from both locations, grain were sampled from 10 randomly chosen heads throughout both rows and combined in a seed envelope. Seed from each plot were surface sterilized by washing for $2 \mathrm{~min}$ in $95 \%$ ethanol followed by a 10-min wash in $1 \%$ sodium hypochlorite with $0.01 \%$ Tween 20 . Five seeds from each plot sample were aseptically placed onto one each of two peptone (Becton, Dickinson and Co. [BD], Sparks, MD) agar media containing either chloramphenicol and the fungicide dichloran (dichloran chloramphenicol peptone agar [DCPA]) (Sigma-Aldrich, St. Louis) (2) or streptomycin (Fisher Scientific, Fair Lawn, NJ) and the fungicide pentacholoronitrobenzene (PCNB) ("Terrachlor"; Uniroyal Co., Middlebury, CT) (40). DCPA is semiselective for Fusarium spp., Alternaria spp., and other dark-spored ascomycetes, while PCNB is semiselective for Fusarium spp. In our experience, using a combination of these two media appears to give a more complete picture of grain colonization by Fusarium and Alternaria spp., because the luxuriant growth of Alternaria spp. on DCPA may overgrow some slower-growing Fusarium spp. Alternatively, Alternaria spp. are nearly completely selected against on PCNB (20). For analysis of grain from single-mutant lines, in 2003, four plots each of each of five genetic backgrounds for wild-type, bmr6, and bmr12 genotypes were grown at two locations so that, in total, 200 seeds of each $b m r$ genotype were screened on each medium. For analysis of grain of double-mutant lines in the background of Wheatland and RTx430 and comparison with single-mutant and wild-type lines, in 2003, four plots of each genotype and each genetic background were planted whereas, in 2004, five plots of each genotype and each genetic background were planted. Thus, in total, 180 seeds of each genotype were screened on each medium.

Each fungal colony growing from an individual seed and onto the agar medium was transferred to one-half strength potato dextrose agar (PDA) (prepared with potato dextrose broth) (BD). $\approx 3$ and $0.1 \%$ of seed had two colonies growing onto DCPA and $\mathrm{PCNB}$, respectively. In these cases, each distinctive colony was transferred to PDA. Fungi were identified using colony morphology on PDA and conidiophore structures and conidial types and morphologies on minimal media $(22,42)$. Putative Fusarium spp. were transferred and grown on $1.5 \%$ agar medium containing $80 \mathrm{mM}$ potassium chloride. Putative Alternaria isolates were transferred by point inoculation to a $2 \%$ water agar medium, followed by aseptic placement of an $\approx 1-\mathrm{cm}^{2}$ filter paper square (Whatman no. 1; Whatman International Ltd., Maidstone, England) over the inoculation site. Fusarium spp. were categorized as members of the G. fujikuroi species complex using nomenclature previously described $(31,44)$. Number of fungi per seed was enumerated in each of the following categories: total fungi, Alternaria spp., Fusarium spp. in the G. fujikuroi species complex, and other Fusarium spp.

The field experimental design was a split plot with whole plots arranged in a randomized complete block, with four replications in 2003 (single-mutant and double-mutant analyses) and five replications in 2004 (double-mutant analyses). Whole plots for single-mutant analyses were genetic background (RTx430, BTx623, BTx631, Redlan, and Wheatland) and subplots were $b m r$ genotype (the wild type, bmr6, and bmr12). Whole plots for double-mutant analyses were genetic background (RTx430 and Wheatland) and $b m r$ genotype (wild-type, bmr6, bmr12, and bmr6, bmr12). Analyses of colonization of grain from singlemutant lines compared with wild-type lines was conducted for each fungal category obtained on each medium. Analyses of colonization of grain from double-mutant lines compared with single-mutant lines and wild-type lines was conducted for each genetic background, for each fungal category, and for each medium. Data were analyzed using Proc Mixed with the following model:

CLASS LOCATION REP GENE LINE;

MODEL DEPENDENT VARIABLE = GENE|LINE;

RANDOM LOCATION REP(LOCATION);

REPEATED/GROUP = LOCATION;

LSMEANS GENE|LINE/PDIFF

Least squares means (LSM) and standard errors (SEs) are reported.

Molecular identification of Fusarium isolates from grain from field-grown $\boldsymbol{b m r}$ and wild-type plants. Molecular identification of representative field isolates was conducted, as described by Geiser and associates (23). This methodology utilizes small but consistent base pair differences in the sequence of the $5^{\prime}$ region of the $T E F$ gene that distinguish each species. The resulting FUSARIUM-ID database has "excellent representation" of species members of G. fujikuroi (23).

To conduct this analysis, isolates grown from grain in one plot and selected on either DCPA or PCNB medium were chosen for further analysis if they had different colony morphologies on PDA or conidial morphology on $\mathrm{KCl}$ and were considered to be representatives of different Fusarium spp. These representative isolates from each plot were single-conidium purified. Isolates collected similarly from a study conducted in 2002 (20) also were included 
in this study. Sequence analyses were conducted on 105 of the 850 isolates collected and morphologically speciated from 2002, 2003, and 2004 field-grown grain.

DNA was extracted from ground lyophilized mycelium $(19,29)$ and the $5^{\prime}$ region of $T E F$ was polymerase chain reaction amplified using primers EF-1 and EF-2 (23). Amplification products were sequenced, and sequences were compared with those in the FUSARIUM-ID database (http://isolate.fusariumdb.org) (23). Among the sequenced isolates were 23 isolates that appeared to be members of the Fusarium incarnatum-F. equiseti species complex (FIESC) as previously defined by O'Donnell and associates in 2007 (45). FUSARIUM-ID and GenBank (4) were used to genotype 22 putative members of FIESC. Two isolates had the greatest similarity (93 to $96 \%$ ) to Fusarium sp. no. 1321, formerly known as Fusarium sp. cf. equiseti, in FUSARIUM-ID. Using GenBank, members of this group, which will be known as FIESC no. 1321 in this study, were found to be most highly similar (92 and $96 \%$ ) in the TEF region to haplotype FIESC 4-a (accession no. EF453007) (45). Seven isolates had the greatest similarity $(\approx 99 \%)$ to $F$. pallidoroseum isolate no. 1320 in FUSARIUM-ID. These isolates, which will be referred to as FIESC no. 1320 for this study, when compared in GenBank, had high similarity to haplotype FIESC 3-a (98 to 99\%) (accession no. EF453042) (45). Thirteen isolates had highest sequence identities (93 to 98\%) to Fusarium sp. no. 1319, formerly known as Fusarium sp. cf. bullatum, in FUSARIUM-ID; members of this group will be called FIESC no. 1319 for this study. When compared in GenBank, these isolates were also most highly similar (91 to 93\%) to FIESC 3-a. Sequences were deposited into GenBank (accession nos. EF152431, GQ339783 to GQ339793, and GU116575 to GU116584).

Due to a high frequency of zeros causing nonnormal distribution of data, and small sample sizes, analyses of Fusarium genotypes collected from single mutant grain (2002 and 2003) was conducted with $\chi^{2}$ tests using PROC FREQ (SAS Institute, Cary, NC). Non-normality of the data was addressed using the EXACT option in PROC FREQ. Quoting SAS (SAS Institute): "Exact tests are appropriate when a data set is small, sparse, skewed, or heavily tied."

Ratios of numbers of each Fusarium genotype obtained from bmr6 or bmrl2 grain was compared with ratios obtained from wild-type grain, which was considered the expected. In most categories of Fusarium spp., genetic background (e.g., RTx430, BTx623, BTx631, Redlan, and Wheatland) was not significant, except for numbers of $F$. thapsinum. Therefore, numbers from each genetic background were combined for analyses. Ratios of Fusarium genotypes colonizing double-mutant grain compared with wild-type grain (2003 and 2004), and single-mutant grain compared with wild-type grain, in two genetic backgrounds (RTx430 and Wheatland) were similarly attempted; however, $\chi^{2} \mathrm{~s}$ from some comparisons approached infinity, presumably due to some Fusarium spp. categories having one or more zeros. Therefore, pairwise comparisons within a Fusarium genotype were conducted; again, comparisons in which both the wild type and bmr genotype had "zeros" were not attempted due to infinite ratios. In this case, by using pairwise analyses, it was established that, within each Fusarium genotype category, there were no significant affects of genetic background. Thus, backgrounds were combined and pooled when making $\chi^{2}$ comparisons of numbers of each Fusarium genotype obtained from bmr6, bmr12, or bmr6, bmrl2 double-mutant grain with those found on wildtype grain, similar to as described above.

Phylogenetic analysis of field isolates and genotypes putatively in FIESC. TEF sequences corresponding to bases 16 to 660 in sequence from Fusarium sp. NRRL 43740 haplotype 3-a (accession no. EF453042) were compared with sequences of seed isolates collected from field studies in 2002, 2003, and 2004 and, presumably, members of FIESC. Also included were FIESC haplotypes 2-a (accession no. EF453019), 3-a, and 4-a (accession no. EF453007); and, from the FUSARIUM-ID database, $F$. pallidoroseum isolate no. 1320 and Fusarium spp. nos. 1319 and 1321. These sequences were chosen because, during BLAST searches, sequences from this study were highly similar to each database sequence. To represent Fusarium spp. outside of FIESC, sequences from $F$. graminearum Schwabe (M02-7035S-2) and $F$. thapsinum (M03-11337S-2), isolated from bmr12 grain, also were included (GenBank accession nos. GU116585 and GU116586). Lengths of sequences were 639 to 651 bases. The DNA sequence alignment was performed using ClustalW function of MacVector (MacVector, Inc., Cary, NC). The unrooted phylogram was constructed using the neighbor-joining method with 1,000 bootstrap replicates; the distances were calculated using the Tamura-Nei algorithm. Gaps distributed proportionally and nodes with bootstrap confidence values $<65 \%$ were collapsed.

Greenhouse inoculations of bmr6, bmr12, and wild-type Redlan plants. The following fungal isolates were used in inoculation studies. F. solani (Mart.) Appel \& Wollenw. emend. Snyd. \& Hans, isolate FRC-M-0900, was obtained from sorghum field soil (Texas). Stalk isolates were $F$. verticillioides (Sacc.) Nirenberg (=F. moniliforme) (isolate FRC-M-1141) (Missouri) and $F$. thapsinum Klittich, Leslie, Nelson et Marasas sp. nov. (isolate FRC-M-3790) (California) (referred to hereafter as F. th.1). These three isolates were obtained from the Fusarium Research Center, Pennsylvania State University. F armeniacum (G. A. Forbes, Windels \& L. W. Burgess) L. W. Burgess \& Summerell (formerly known as $F$. acuminatum) (isolate H02781L-5b) and FIESC no. 1320 (formerly known as $F$. pallidoroseum or F. semitectum) (isolate M03-11261L-9) were isolated from sorghum leaves (Nebraska). F. thapsinum (isolate H03-11-9) (21) (referred to hereafter as F. th.-2) and Alternaria alternata (Fr.:Fr.) Keissl. (isolate H02-755S) (20) were obtained from sorghum grain (Nebraska). Species or genotype identifications were confirmed using sequence analysis of conserved DNA regions (GenBank accession nos. EF152426, EF152427, EF152428, EF152433, EU016680, and GU116571 to GU116574). Inocula were prepared by inoculating sterile toothpicks in $5 \mathrm{ml}$ of sterile potato dextrose broth with a mycelium-covered agar plug from a PDA plate grown for 7 days. The broth-and-toothpick cultures were incubated for 2 weeks at $25^{\circ} \mathrm{C}$ prior to inoculations (25).

For greenhouse inoculations, plants of cv. Redlan near isogenic for $b m r 6, b m r 12$, and the wild type were grown by sowing seed into 25.4-cm-diameter pots containing standard soil mix. Seedlings were culled to two per pot; two plants of the same genotype were in each pot in order to utilize greenhouse space more efficiently while reducing the opportunity for error that would be associated with seeding multiple genotypes in a single pot. $\mathrm{Cv}$. Redlan was chosen because, in a previous study, lesions on wildtype plants were intermediate in length following inoculation with F. th.-1 compared with lesions produced on five other genotypes (20). Peduncles of plants 2 weeks after anthesis (defined as approximately half the anthers exerted) were inoculated with fungal-infested toothpicks or toothpicks incubated in sterile broth (25). Plants within a single pot were inoculated with two different fungi or a fungal culture and broth control. Eighteen days following inoculation, peduncles were split longitudinally and the length of the discolored region was measured. This measurement will be referred to as "lesion length."

The experimental design consisted of six replications containing 12 pots each, with each pot containing two plants. Individual plants were considered the experimental units. Within each replication, each of three $b m r$ genotypes was randomly assigned to four pots and one of eight inoculation treatments assigned to each plant so that each $b m r$-inoculation treatment combination occurred once per replication. Pairings of inoculation treatments within pots were randomized for each replication. Replications were blocked by location within the greenhouse. The experiment 
(set of six replications) was repeated thrice across time. When the three repetitions of the experiment were combined for analyses, there were significant time period and treatment-time period effects. Therefore, LSMs for each repetition were analyzed separately using PROC MIXED with the following model:

\section{CLASS ENVIRON REP BMR INOCULUM;}

MODEL DEPENDENT VARIABLE = BMR|INOCULUM;

RANDOM REP POT(REP);

\section{LSMEANS BMR|INOCULUM/DIFF;}

LSMs and SEs are reported.

During the third repetition of the experiment, portions of each peduncle were analyzed for fungal growth. After measuring lesion lengths from plants in each block, one-half of a longitudinally split peduncle was surface sterilized in $1 \%$ sodium hypochlorite and rinsed in sterile distilled water, three times. Workers changed disposable gloves and sprayed gloved hands with $70 \%$ ethanol before handling each peduncle. Using surface-sterilized scalpels or razor blades on a surface-sterilized tray, the following sections were cut: $1-\mathrm{cm}$ long sections measured $1 \mathrm{~cm}$ above and below the lesion; 1-cm long sections including the edges of the lesion; and, for lesions larger than $1 \mathrm{~cm}$, one or two additional sections up to $1 \mathrm{~cm}$ long each from within the lesion. Sorghum is a silicaceous plant (35) and slicing the peduncle crosswise could cause it to break apart; thus, the size and number of cut sections obtainable were limited. Position along the peduncle (e.g., above the inoculation site toward the head or below the inoculation site toward the flag leaf) was recorded. If only one within-lesion section was dissected, it included the inoculation site. Using surface-sterilized forceps, the pieces were transferred to $100 \mathrm{~mm}$ square style petri dishes with 13-mm gridlines (BD Falcon; BD Biosciences, San Jose, CA) prepared with $35 \mathrm{ml}$ of DCPA medium. Plated peduncle sections were incubated at room temperature for 3 days, then scored "+" or "-" for fungal growth onto the agar medium. A $\chi^{2}$ analysis of percentage of sections with fungal growth from each position along the peduncle and for each $b m r$ genotype (bmr6 and bmr12) were compared with percentage of wild-type sections with fungal growth using PROC FREQ. Results from all fungal treatments were combined. The possibility of positional effects was tested by comparing comparable sections from above the inoculation site versus below the inoculation site; fungus-inoculated peduncles were considered separately from those that were broth inoculated. In only one instance (withinlesion sections from fungal-inoculated wild-type peduncles) was there a positional effect $(P=0.03)$. For ease of analysis, comparable sections (e.g., outside the lesions, lesion borders, and within lesions) were combined for analysis. Two-sided $P \leq 0.05$ were considered significant.

\section{RESULTS}

Fusarium and Alternaria spp. colonization of field-grown grain from $b m r$ and wild-type plants. Colonization of grain from bmr6, bmr12, and near-isogenic wild-type plants (grown in 2003) was analyzed by selecting fungi grown from random seed from each sample on PCNB and DCPA media. Across genetic backgrounds, bmr genotypes had a significant effect on total numbers of colonies when selected on either medium $(P \leq 0.02)$ (Table 1). The bmr genotype also had a significant effect on numbers of $G$. fujikuroi isolates per seed when selected on PCNB $(P=0.04)$; isolates per grain from $b m r 12$ plants were significantly less than those from wild-type seed (Table 1). When selected on DCPA medium, bmr genotype had a significant effect on $G$. fujikuroi isolates per seed for colonization of bmr6 grain when compared with wild-type plants $(P=0.02)$. Numbers of Alternaria spp. selected on DCPA per seed in bmrl2 grain were significantly fewer than those from $b m r 6$ grain $(P=0.03)$. There was no apparent significant effect of $b m r$ genotype on numbers of other Fusarium spp. in this analysis when fungi grown from seed were selected on either medium (Table 1).

Fungal colonies growing from field-grown grain of bmr6, bmr12 double-mutant plants, as well as bmr6, bmr12, and wildtype plants in the genetic backgrounds Wheatland and RTx430 (2003 and 2004), also were selected on DCPA or PCNB (Table 2). In this analysis, there was no indication that combining mutations had a greater effect than each individual mutation in near-isogenic lines on colonization by the fungi assessed in this study. When selected on DCPA, grain from $b m r$ genotypes in the background Wheatland had significantly fewer G. fujikuroi isolates than the wild type while selection on PCNB of RTx430 bmr12 grain resulted in significantly fewer $G$. fujikuroi isolates compared with other $b m r$ genotypes in this background (Table 2). The $b m r$ genotype did not have a significant effect on colonization of grain by other Fusarium spp. in either background. However, significantly more other Fusarium spp. were recovered on PCNB medium from Wheatland $b m r 12$ grain than from RTx430 bmrl2 grain $(P=0.04)$ (Table 2). When considering numbers of Alternaria spp., colonies per seed selected on DCPA were significantly higher in wild-type RTx430 grain than from near-isogenic bmr12 and bmr6, bmrl2 double-mutant grain; however, differences in colonization of wild-type and $b m r$ grain in Wheatland background were not significant (Table 2). Fusarium spp. obtained from wildtype grain, as determined by morphological characters (42), were $F$. moniliforme (e.g., unable to distinguish whether $F$. verticillioides or $F$. thapsinum), F. proliferatum (Matsush.) Nirenberg ex Gerlach \& Nirenberg, F. solani, F. subglutinans (Wollenw. \& Reinking) P. E. Nelson, F. thapsinum, F. verticillioides, and unidentified Fusarium spp. Fusarium spp. obtained from bmr6 grain were similar to those from wild-type grain, except no $F$. proliferatum isolates were identified morphologically. Fusarium spp. isolated from bmrl2 grain and identified by morphological characteristics were $F$. aquaeductuum (Radlk. \& Rabenh.) Lagerh., $F$. moniliforme, $F$. proliferatum, $F$. verticillioides, and unidentified Fusarium spp. As determined by morphological characteristics, F. avenaceum (Fr.) Sacc., F. equiseti (Corda) Sacc.

TABLE 1. Number of fungal colonies per seed of wild-type, brown midrib $(b m r) 6$, and bmr12 plants grown at two locations and screened on two semiselective media

\begin{tabular}{lccccc}
\hline & & \multicolumn{4}{c}{ Number of colonies per seed $^{\mathrm{z}}$} \\
\cline { 3 - 6 } bmr genotype & Medium & Total colonies & Alternaria spp. & Gibberella fujikuroi $^{\mathrm{y}}$ & Other Fusarium spp. \\
\hline Wild type & DCPA & $0.92 \mathrm{a} \pm 0.07$ & $0.72 \mathrm{ab} \pm 0.05$ & $0.09 \mathrm{a} \pm 0.03$ & $0.02 \mathrm{a} \pm 0.01$ \\
bmr6 & DCPA & $0.94 \mathrm{a} \pm 0.07$ & $0.74 \mathrm{a} \pm 0.05$ & $0.02 \mathrm{~b} \pm 0.03$ & $0.01 \mathrm{a} \pm 0.01$ \\
bmr12 & DCPA & $0.75 \mathrm{~b} \pm 0.07$ & $0.61 \mathrm{~b} \pm 0.05$ & $0.05 \mathrm{ab} \pm 0.03$ & $0.02 \mathrm{a} \pm 0.01$ \\
Wild type & PCNB & $0.15 \mathrm{a} \pm 0.05$ & na & $0.09 \mathrm{a} \pm 0.03$ & $0.01 \mathrm{a} \pm 0.01$ \\
bmr6 & PCNB & $0.06 \mathrm{~b} \pm 0.05$ & na & $0.06 \mathrm{ab} \pm 0.03$ & $0.01 \mathrm{a} \pm 0.01$ \\
bmr12 & PCNB & $0.08 \mathrm{ab} \pm 0.05$ & na & $0.04 \mathrm{~b} \pm 0.03$ & $0.02 \mathrm{a} \pm 0.01$ \\
\hline
\end{tabular}

y Dichloran chloramphenicol peptone agar (DCPA) is semiselective for Fusarium spp., Alternaria spp., and other dark-spored ascomycetes and pentachloronitrobenzene (PCNB) medium is semiselective for Fusarium spp.

${ }^{\mathrm{z}}$ Least squares means and standard errors are shown. Within each category of fungal counts and for each medium, values with differing letters are statistically significant at $P \leq 0.05$; na indicates not applicable because PCNB is strongly selective against Alternaria spp. 
sensu Gordon, F. moniliforme, F. proliferatum, F. thapsinum, $F$. verticillioides, and unidentified Fusarium spp. were recovered from grain of double-mutant plants.

Molecular identification of Fusarium isolates from grain from field-grown $\boldsymbol{b m r}$ and wild-type plants. Sequence analysis of the 5' TEF region from representative Fusarium isolates from single-mutant bmr genotypes and near-isogenic wild-type genotypes (2003) and from a previously reported study (2002) (20) were genotyped by comparison with sequences of type isolates in FUSARIUM-ID. Eleven different species or genotypes were distinguished. The most frequently isolated from wild-type grain were $F$. thapsinum, FIESC no. 1319, and F. proliferatum. Other commonly isolated species or genotypes recovered from wildtype grain were FIESC no. $1320, F$. verticillioides, $F$. graminearum, and $F$. subglutinans. Four species were recovered infrequently (from one or two plots) from these trials. The distribution of fungal isolates recovered from wild-type, bmr6, and bmr12 grain is illustrated in Figure 1. The $\chi^{2}$ analyses, using ratios of Fusarium spp. isolated from wild-type grain as "expected," showed that numbers of each genotype found on bmrl2 were significantly different (exact $\chi^{2}, P=0.05$ ). Further analyses, comparing numbers of each species or genotype found on bmrl2 grain with that found on wild-type grain, showed that the difference could be attributed, in part, to numbers of FIESC no. $1319(P<0.01)$, which was the second most frequently isolated Fusarium sp. from wild-type grain but was not detected in $b m r 12$ grain. Colonization by $F$. proliferatum also was reduced in $b m r 12$ grain compared with the wild type $(P=0.06)$.

Sequence comparisons between $5^{\prime} T E F$ regions from isolates representing Fusarium spp. obtained from bmr6, bmr12 doublemutant grain also were made with those from single-mutant $b m r 6$ and bmrl2 and wild-type Wheatland and RTx430 plants (2003 and 2004). F. thapsinum was the predominant species isolated and there were no significant differences detected in this analysis for colonization by $F$. thapsinum of wild-type, bmr6, bmr12, or bmr6, bmrl2 grain $(P \geq 0.14)$. Other species isolated from wild-type and bmr6 grain included $F$. proliferatum, $F$. verticillioides, and FIESC no. 1320. Surprisingly, none of these other species were recovered from bmrl2 grain and only FIESC no. 1320 was found in bmr6, bmr12 double-mutant grain, whereas FIESC genotype no. 1321 was isolated only from $b m r 12$ and double-mutant grain in RTx430 and Wheatland genetic backgrounds. Pairwise comparisons for each fungal species showed there were no significant differences of numbers isolated from each bmr genotype compared with those isolated from wild-type grain $(P \geq 0.25)$.

Phylogenetic analysis of field isolates and genotypes putatively in FIESC. When phylogenetic analysis was performed on TEF sequences from putative FIESC members obtained from sorghum grain during this study, results indicated that one genotype, represented by a clade, was present in bmr6 and wildtype grain but not in $b m r 12$ grain. $T E F$ sequences were compared with sequences of FIESC haplotypes; representative isolates of $F$. pallidoroseum (no. 1320), Fusarium sp. cf. equiseti (no. 1321), and Fusarium sp. cf. bullatum (no. 1319) from FUSARIUM-ID; and $F$. graminearum and $F$. thapsinum isolates obtained from bmr12 grain (Fig. 2). All the sorghum grain isolates that were putatively identified as FIESC were in the larger clade that includes haplotypes of FIESC 2-a, 3-a, and 4-a and F. pallidoroseum, Fusarium sp. cf. equiseti, and Fusarium sp. cf. bullatum, and separately from $F$. graminearum and $F$. thapsinum, indicating that they were, indeed, members of FIESC. This large clade separated into three branches. One branch had a small clade that included haplotypes FIESC 2-a and 4-a and Fusarium sp. cf.

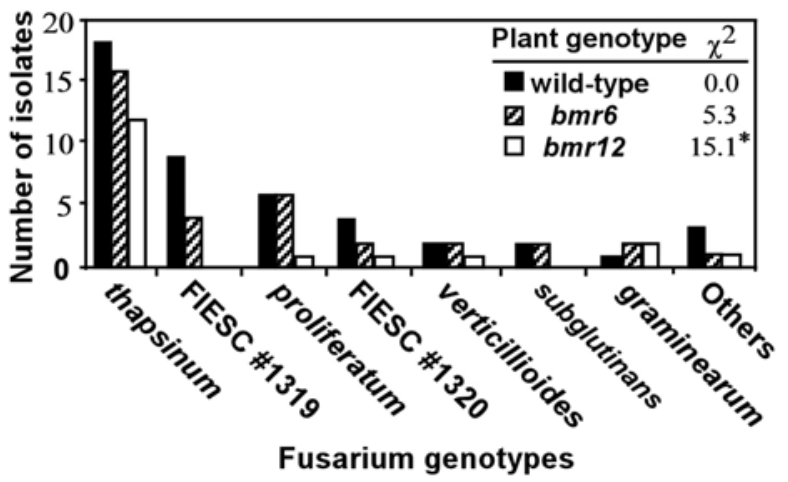

Fig. 1. Distribution of Fusarium genotypes isolated from wild-type and nearisogenic brown midrib (bmr)6 and bmr12 grain. DNA from representative Fusarium grain isolates collected from each field plot were used for sequence analysis of polymerase chain reaction fragments from a region of the translation elongation factor 1- $\alpha$ gene; sequences were compared with those in the FUSARIUM-ID database. A $\chi^{2}$ analysis of ratios of the genotypes found in bmr6 and bmr12 grain were compared with those in wild-type grain; "*" indicates that $\chi^{2}$ is significant $(P=0.05)$. FIESC $=$ Fusarium incarnatum- $F$. equiseti species complex.

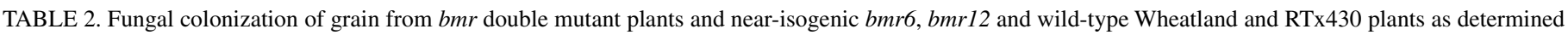
by selecting on two media

\begin{tabular}{|c|c|c|c|c|c|c|}
\hline \multirow[b]{2}{*}{ Medium ${ }^{y}$} & \multirow[b]{2}{*}{ Plant background } & \multirow[b]{2}{*}{$b m r$ Genotype } & \multicolumn{4}{|c|}{ Number of colonies per seed ${ }^{\mathrm{z}}$} \\
\hline & & & Total colonies & Alternaria spp. & Gibberella fujikuroi & Other Fusarium spp. \\
\hline DCPA & Wheatland & Wild-type & $0.95 \mathrm{ab} \pm 0.08$ & $0.80 \mathrm{ab} \pm 0.06$ & $0.05 \mathrm{a} \pm 0.03$ & $0.01 \mathrm{a} \pm 0.01$ \\
\hline DCPA & Wheatland & bmr6 & $0.99 \mathrm{a} \pm 0.08$ & $0.84 \mathrm{a} \pm 0.06$ & $0.03 \mathrm{~b} \pm 0.03$ & $0.01 \mathrm{a} \pm 0.01$ \\
\hline DCPA & Wheatland & bmr12 & $0.81 \mathrm{~b} \pm 0.08$ & $0.68 \mathrm{~b} \pm 0.06$ & $0.03 \mathrm{~b} \pm 0.03$ & $0.01 \mathrm{a} \pm 0.01$ \\
\hline DCPA & Wheatland & bmr6, bmr12 & $0.89 \mathrm{ab} \pm 0.08$ & $0.78 \mathrm{ab} \pm 0.06$ & $0.03 \mathrm{~b} \pm 0.03$ & $0.02 \mathrm{a} \pm 0.01$ \\
\hline DCPA & RTx430 & Wild-type & $0.84 \mathrm{a} \pm 0.08$ & $0.68 \mathrm{a} \pm 0.06$ & $0.03 \mathrm{a} \pm 0.03$ & $0.01 \mathrm{a} \pm 0.01$ \\
\hline DCPA & $\mathrm{RTx} 430$ & bmr6 & $0.72 \mathrm{a} \pm 0.08$ & $0.60 \mathrm{ab} \pm 0.06$ & $0.03 \mathrm{a} \pm 0.03$ & $0.01 \mathrm{a} \pm 0.01$ \\
\hline DCPA & $\mathrm{RT} 4430$ & bmr12 & $0.39 \mathrm{~b} \pm 0.08$ & $0.32 \mathrm{c} \pm 0.06$ & $0.03 \mathrm{a} \pm 0.03$ & $0.01 \mathrm{a} \pm 0.01$ \\
\hline DCPA & $\mathrm{RTx} 430$ & bmr6, bmr12 & $0.48 b \pm 0.08$ & $0.39 b c \pm 0.06$ & $0.03 \mathrm{a} \pm 0.03$ & $0.01 \mathrm{a} \pm 0.01$ \\
\hline PCNB & Wheatland & Wild-type & $0.06 \mathrm{a} \pm 0.05$ & na & $0.04 \mathrm{a} \pm 0.04$ & $0.01 \mathrm{a} \pm 0.01$ \\
\hline PCNB & Wheatland & bmr6 & $0.08 \mathrm{a} \pm 0.05$ & na & $0.06 \mathrm{a} \pm 0.04$ & $0.02 \mathrm{a} \pm 0.01$ \\
\hline PCNB & Wheatland & bmrl2 & $0.08 \mathrm{a} \pm 0.05$ & na & $0.05 \mathrm{a} \pm 0.04$ & $0.04 \mathrm{a} \pm 0.01$ \\
\hline PCNB & Wheatland & bmr6, bmr12 & $0.09 \mathrm{a} \pm 0.05$ & na & $0.02 \mathrm{a} \pm 0.04$ & $0.02 \mathrm{a} \pm 0.01$ \\
\hline PCNB & $\mathrm{RT} \times 430$ & Wild-type & $0.11 \mathrm{a} \pm 0.05$ & na & $0.08 \mathrm{a} \pm 0.04$ & $0.01 \mathrm{a} \pm 0.01$ \\
\hline PCNB & $\mathrm{RTx} 430$ & bmr6 & $0.07 \mathrm{ab} \pm 0.05$ & na & $0.06 \mathrm{ab} \pm 0.04$ & $0.00 \mathrm{a} \pm 0.01$ \\
\hline PCNB & $\mathrm{RT} \times 430$ & bmr12 & $0.04 \mathrm{~b} \pm 0.05$ & na & $0.03 \mathrm{~b} \pm 0.04$ & $0.00 \mathrm{a} \pm 0.01$ \\
\hline PCNB & $\mathrm{RT} \times 430$ & bmr6, bmr12 & $0.05 b \pm 0.05$ & na & $0.04 \mathrm{ab} \pm 0.04$ & $0.00 \mathrm{a} \pm 0.01$ \\
\hline
\end{tabular}

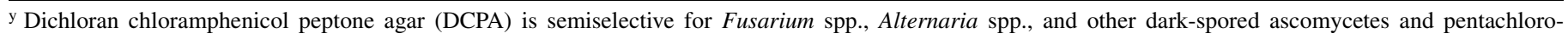
nitrobenzene (PCNB) medium is semiselective for Fusarium spp.

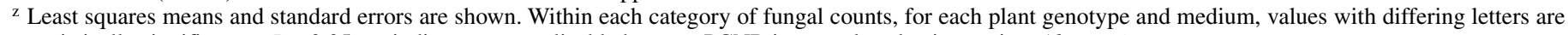
statistically significant at $P \leq 0.05$; na indicates not applicable because PCNB is strongly selective against Alternaria spp. 
equiseti. Another branch, which included all sorghum FIESC isolates but two, split into two clades. Interestingly, one clade had isolates only from the wild type and bmr6 (6). The other clade included Fusarium sp. cf. bullatum, haplotype FIESC 3-a, and F. pallidoroseum and grain isolates from wild-type, bmr6, and bmr12 plants. The third branch within the larger clade included only two isolates, both from $b m r 12$ plants. This analysis indicates that a previously uncharacterized haplotype of FIESC existed, which preferentially colonized wild-type and bmr6 grain but not bmrl2 grain.

Greenhouse inoculations of bmr6, bmr12, and wild-type Redlan plants. Three repetitions of an assay in which peduncles of plants were inoculated with different fungi were conducted. These experiments indicated that, when near-isogenic bmr6, bmr12, and wild-type plants were inoculated by $F$. verticillioides or A. alternata, as well as two $F$. thapsinum isolates, the mean lesion lengths could be significantly shorter on $b m r$ plants than those resulting on wild-type plants. Lesions (areas of discoloration) produced on peduncles of bmr6, bmrl2, and wild-type plants in cv. Redlan background following inoculation with one of two $F$. thapsinum isolates; with an isolate of $F$. verticillioides, $F$. armeniacum, F. solani, FIESC no. 1320, or A. alternata; or with sterile broth were compared in three repetitions. Because of significant effects, each repetition of the experiment was considered independently. For the first repetition, $b m r$ genotype, inoculum, and their interactions were significant $(P \leq 0.04)$.
Inoculations of wild-type plants by either $F$. thapsinum isolate, $F$. verticillioides, $F$. solani, $F$. armeniacum, or A. alternata resulted in mean lesion lengths significantly greater than the discoloration resulting from broth inoculation (Fig. 3A). Mean lesion lengths produced on $b m r$ plants by F. th.-2, F. solani, F. armeniacum, A. alternata, or $F$. verticillioides (bmr6 plants only) were significantly smaller than those produced on wild-type plants. During the second repetition of the experiment, only inoculum was significant $(P<0.01)$; although inoculation of wild-type plants by F. th.-2 and $F$. verticillioides resulted in mean lesion lengths significantly greater than discoloration resulting from broth inoculation, there were no significant differences between mean lesion lengths produced on wild-type and bmr plants (Fig. 3B).

During the third repetition of the experiment, results were significant for $b m r$ genotype, inoculum, and their interactions $(P \leq 0.03)$. Mean lesion lengths resulting from inoculation of wild-type plants with either $F$. thapsinum isolate, $F$. verticillioides, $F$. armeniacum, and $A$. alternata were significantly greater than discoloration resulting from inoculation with broth (Fig. 3C). Inoculation of both bmr genotypes by $\mathrm{F}$. th.-1 and $F$. verticillioides, or bmr6 plants with F. th.-2 and A. alternata, yielded mean lesion lengths significantly less than those following inoculation of the same fungi onto wild-type plants (Fig. 3C). To provide an indication of the extent of fungal growth in peduncles of inoculated plants, $1-\mathrm{cm}$ sections from peduncles were plated and assessed for growth (+ or -) on DCPA medium. Percentage of

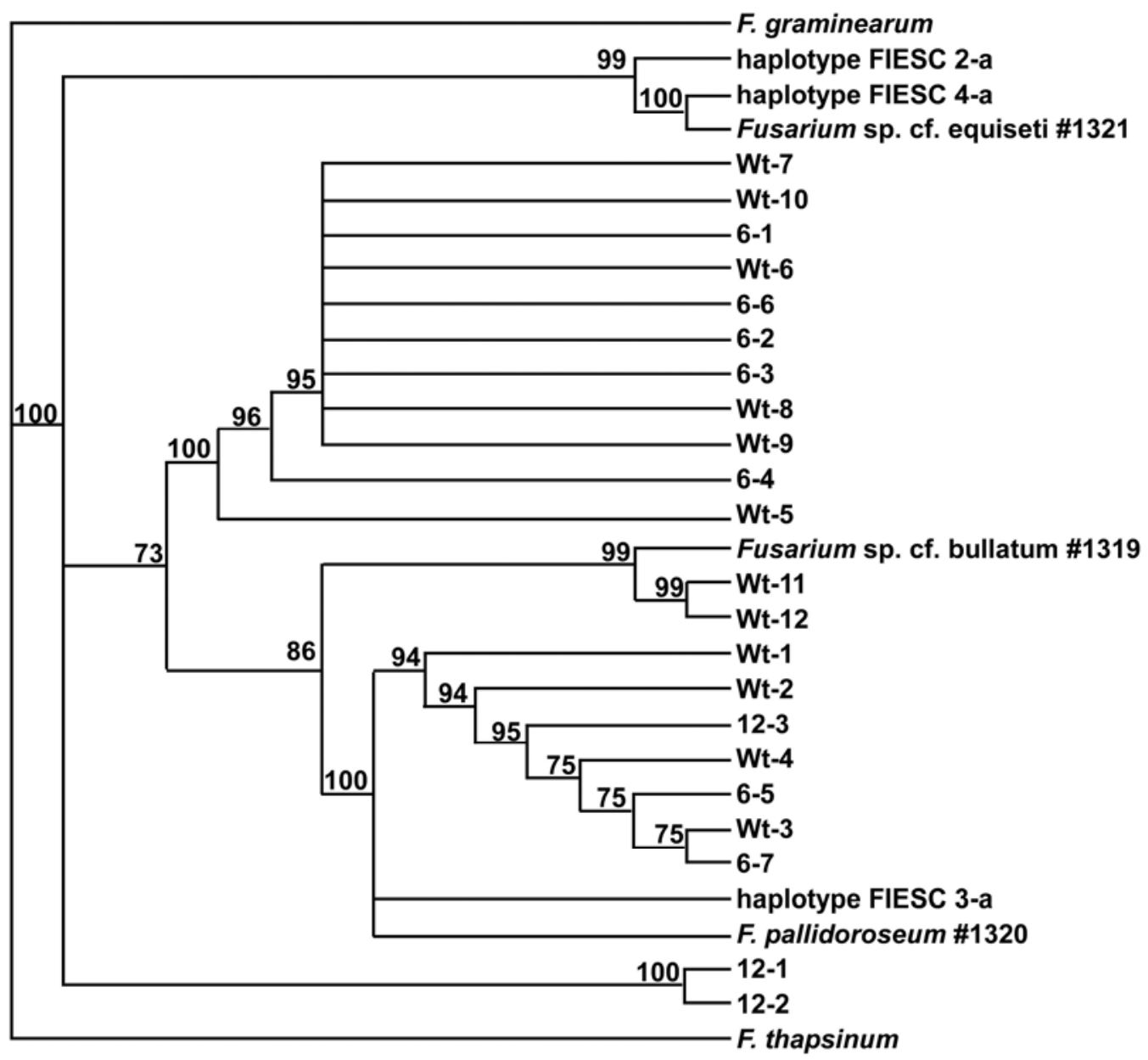

Fig. 2. Phylogenetic analysis of sequences of a portion of translation elongation factor (TEF) 1- $\alpha$ gene from putative Fusarium incarnatum-F. equiseti species complex (FIESC) isolates from sorghum grain, type isolates from FUSARIUM-ID database, and haplotypes from GenBank (see Materials and Methods). FIESC isolates were isolated from grain from 2002, 2003, and 2004 field studies. "Wt" indicates an isolate from wild-type grain, "6" indicates an isolate from brown midrib (bmr)6 grain, and "12" indicates an isolate from bmrl2 grain. Isolates nos. 1319, 1320, and 1321 and haplotypes 2-a, 3-a, and 4-a had greatest similarity to isolates from this study when BLAST was conducted in FUSARIUM-ID or GenBank databases, respectively. TEF sequences from $F$. graminearum and $F$. thapsinum, isolated from bmrl2 grain, were included as outgroups. Bootstrap values (percentage) for 1,000 replicates are indicated by each node. 
peduncle sections from outside the lesion, at lesion borders, or within the lesion for bmr6 or bmrl2 plants inoculated with fungi were compared with those from wild-type plants (Table 3 ). Fungal growth from bmr6 peduncle sections was not significant but sections from borders and from within lesions of bmrl2 peduncles had significantly greater fungal growth compared with lesions on wild-type peduncles.

\section{DISCUSSION}

In numerous studies, lignin has been implicated in defense against pathogens and has been proposed as a physical barrier against penetration, further ingress, or diffusion of pathogenproduced toxins $(8,17)$. Although induction of lignin synthesis is likely to be part of a generalized resistance response (43), the results presented herein suggest that impairing lignin biosynthesis does not lessen resistance to some pathogens. In particular, we propose that bmrl2 may modify phenylpropanoid metabolism including and beyond lignin biosynthesis, thus restricting or excluding growth of a genotype of FIESC from grain of these plants.

In a previous study, we showed that, when grain from two genetic backgrounds (RTx430 and Wheatland) grown at two locations was plated onto PCNB agar medium, numbers of other Fusarium spp. could be significantly reduced in bmr genotypes; numbers of $G$. fujikuroi isolates also could be significantly reduced but there appeared to be an effect of environment on colonization of grain (20). Even when plating larger numbers of grain from a given genetic background grown in different environments onto DCPA medium, the results for Fusarium spp. were less clear. We concluded that plating sorghum grain onto both media, side by side, which was done in the current study, appeared to present a more complete, if not more complicated, picture of infection or colonization of the grain mold fungi Alternaria and Fusarium spp. The modes of action of dichloran (in DCPA) or PCNB have not been reported. Tolerance of PCNB by Fusarium spp. was proposed to be due to a combination of reduced uptake as well as metabolism to less toxic compounds (39). PCNB appears to affect specific activity of glutathione-Stransferase in Fusarium spp. (12) as well as DNA excision repair in bacteria (11). In the present study, results from plating grain on both DCPA and PCNB to screen fungal growth from grain supported the contention that $b m r$ genotype effects colonization by members of the $G$. fujikuroi species complex across genetic backgrounds (Table 1).

Representative Fusarium isolates from each plot from this and the previous study were genotyped by sequence analysis of a portion of the $T E F$ gene, then compared with sequences from type isolates in the FUSARIUM-ID database. We were surprised to find that one genotype, having high similarities to FIESC isolate
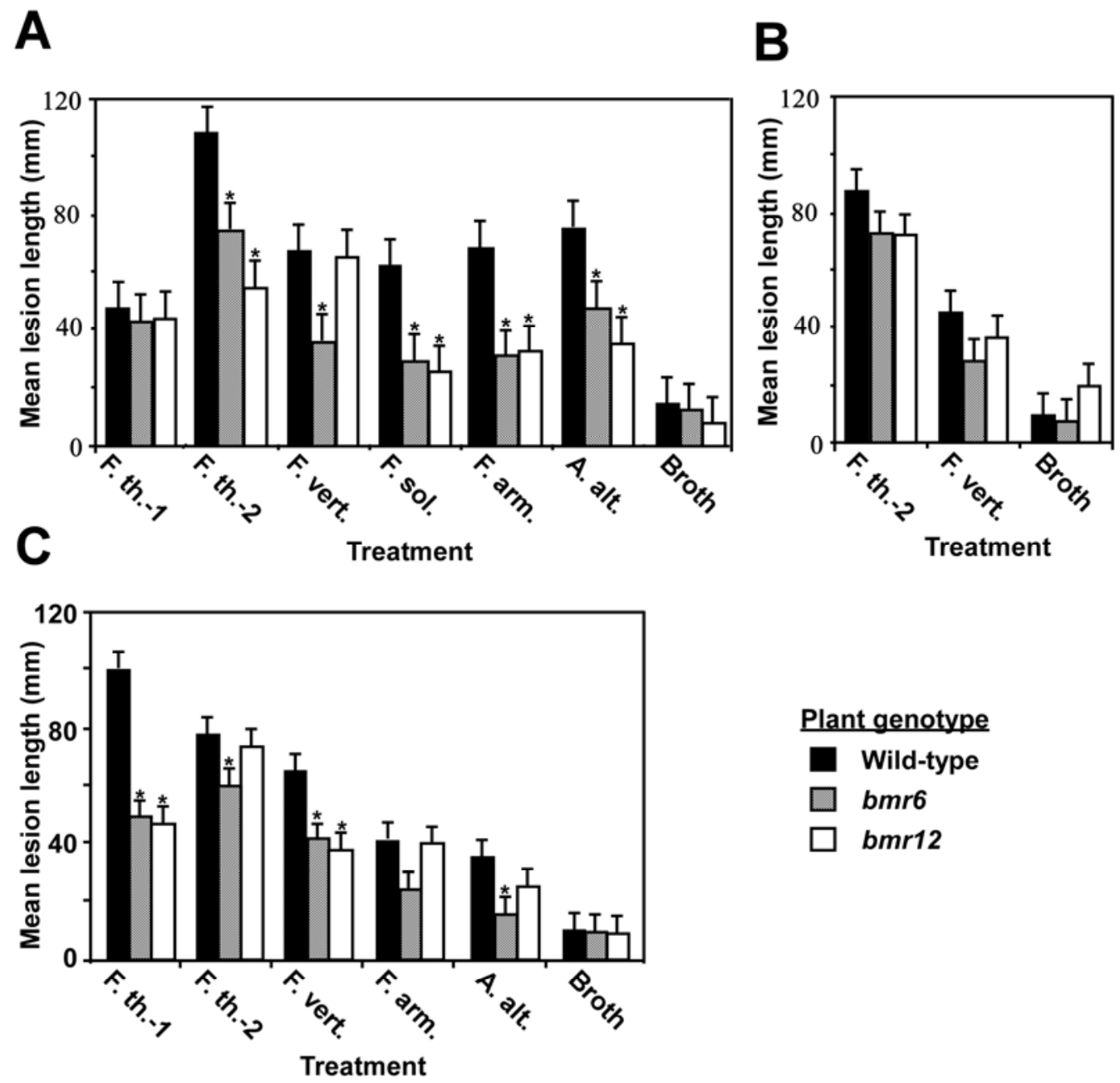

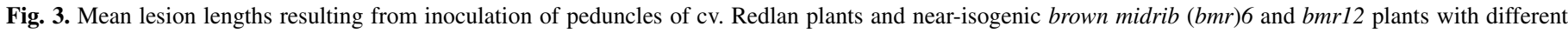

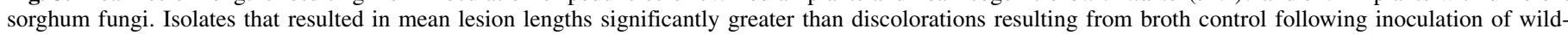

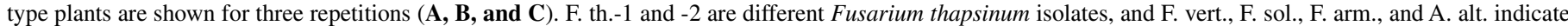

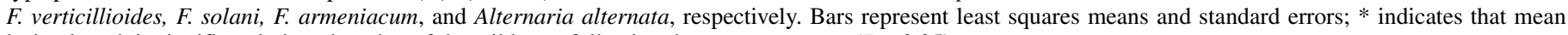
lesion length is significantly less than that of the wild type following the same treatment $(P \leq 0.05)$. 
no. 1319, did not appear to colonize bmrl2 grain across plant genetic backgrounds. Several FIESC isolates from wild-type or bmr6 grain were most similar to genotype no. 1319, formerly known as Fusarium sp. cf. bullatum; therefore, for this study, they were referred to as FIESC no. 1319. Two of these isolates, from different locations in 2 years, had $98 \%$ similarity to FIESC no. 1319 (Wt-11 and Wt-12) (Fig. 2). The rest of the isolates had 93\% similarity to FIESC no. 1319 (Wt-6 through Wt-10, 6-1 through 6-4, and 6-6) and phylogenetic analysis indicated that these isolates, which apparently group within the FIESC, also group separately from genotype no. 1319 and from the three FIESC haplotypes most similar to sequences from the present study (2-a, 3-a, and 4-a) (45), indicating a previously unreported haplotype within FIESC. This new haplotype, isolated from wild-type and bmr6 grain, was excluded from colonizing bmrl2 grain in our experiments (Figs. 1 and 2). Members of FIESC, identified morphologically in this study as $F$. pallidoroseum (also known as F. semitectum), F. equiseti, or simply Fusarium spp., probably colonize sorghum grain asymptomatically (47). Inoculation of peduncles with an FIESC no. 1319 isolate resulted in mean lesion lengths that were not significantly different from the broth control, while inoculation of developing flowers with the same isolate resulted in mature grain that was as healthy as uninoculated controls, as indicated by three measurements (22). In the present study, an FIESC no. 1320 isolate inoculated onto wildtype plants in three repetitions had mean lesion lengths not significantly different from those of the broth control (Fig. 3). These results suggest that at least some genotypes in FIESC are low in virulence on sorghum. However, FIESC isolates may cause deterioration of stored grain (38) or produce mycotoxins $(27,63)$. In addition to apparently excluding at least one member of FIESC, evidence suggests that colonization by $F$. proliferatum, which is relatively common in wild-type and bmr6 grain, also was limited in bmrl2 grain (Fig. 1). F. proliferatum is a member of species complexes that are known to cause grain mold and stalk rot diseases and can produce mycotoxins which reduce the quality and usability of sorghum grain $(43,58,59)$.

TABLE 3. Percent of sections from fungal-inoculated peduncles having fungal growth as selected on dichloran chloramphenicol peptone agar (DCPA) medium $^{\mathrm{x}}$

\begin{tabular}{lccc}
\hline & \multicolumn{3}{c}{ Plant genotype $^{\mathrm{z}}$} \\
\cline { 2 - 4 } Peduncle section $^{\mathrm{y}}$ & Wild-type & bmr6 & bmr12 \\
\hline Outside lesion & $40.5(79)$ & $48.8(84)$ & $46.0(76)$ \\
Lesion borders & $82.0(78)$ & $79.8(84)$ & $90.8^{*}(76)$ \\
Within lesion & $88.3(60)$ & $93.1(58)$ & $100.0^{*}(44)$ \\
\hline
\end{tabular}

${ }^{x}$ Peduncles inoculated with either Fusarium thapsinum (two isolates), $F$. verticillioides, $F$. solani, $F$. armeniacum, $F$. incarnatum- $F$. equiseti species complex no. 1320, or Alternaria alternata isolates, generated during the third repetition, were sectioned and plated to estimate extent of fungal growth. For each location along the peduncle, $\chi^{2}$ analysis of percentage of sections with fungal growth for each $b m r$ genotype (bmr6 and $b m r 12)$ were compared with percentage of wild-type sections with fungal growth using PROC FREQ (SAS).

${ }^{y}$ Following measurement of lesions, peduncles from the third repetition were dissected (see Materials and Methods). From each peduncle, sections $1 \mathrm{~cm}$ above and below the lesion, 1-cm sections that included the upper or lower border of the lesion, and, if the lesion was $>10 \mathrm{~mm}, 1-\mathrm{cm}$ sections from within the lesion were cut and aseptically placed onto DCPA medium. Three days after plating, each section was scored for fungal growth onto the medium. Percentage of peduncle pieces with fungal growth are reported. For peduncles inoculated with broth, outside lesion sections from bmr6 plants had significantly more fungal growth $(33.3 \%)$ compared with wild-type plants $(0.0 \%)(P<0.01)$. There were no significant differences in fungal growth from boundary sections from broth-inoculated lesions. There were too few "within-lesion" sections (lesions $>1 \mathrm{~cm}$ ) for comparison.

${ }^{\mathrm{z}}$ Numbers in parentheses indicate number of sections tested. Percent growth from peduncle sections from each wild-type plant was compared with that from comparable sections from each similarly-treated $b m r$ plant; * indicates percentages are significantly different from the wild type at $P \leq 0.05$.
Our previous study indicated that $b m r$ grain had reduced colonization by Fusarium spp. and, when peduncles were inoculated with a virulent $F$. thapsinum isolate, mean lesion lengths on bmr plants were reduced compared with near-isogenic genotypes when six genetic backgrounds were compared (20). Other studies in which reduced lignin did not result in increased susceptibility to necrotrophic fungi have been conducted in smooth bromegrass and soybean. In the case of smooth bromegrass, clones with reduced lignin were more susceptible to a biotrophic fungus but not to two necrotrophic fungi (15). In soybean, it was shown that, among six cultivars with different levels of resistance to Sclerotinia stem rot, lignin content was positively correlated to disease severity (51). In the present study, it is likely that the reducedlignin phenotypes in the near-isogenic $b m r$ genotypes were due to defective genes in the lignin biosynthesis pathway $(5,48,56)$. In general, these mutations have resulted in an overall reduction in colonization by G. fujikuroi. However, bmrl2 also appeared to be selective against one or two Fusarium genotypes. Resistance genes that encode receptor proteins, which interact with specific microorganism-produced extracellular molecules (effectors) in a gene-for-gene manner, can block a potentially pathogenic genotype from infecting its host (9). However, in the present study, the bmrl2 gene encoded for an impaired enzyme in the lignin biosynthetic pathway $(9,57)$.

Biochemically, it is known that the $b m r$ plants sampled for this study had reduced lignin content (as measured by acid detergent concentration [ADL]); at least one of the $b m r$ genotypes from each plant background had significantly reduced ADL compared with that of its wild-type counterpart (46). In particular, both bmr6 and bmr12 lines in the Redlan background and another background commonly used in breeding, Wheatland, had significantly reduced ADL. Lignin pathway intermediates also were measured in bmr genotypes in RTx430 and Wheatland backgrounds (48). Genetic background appeared to interact with each bmr gene and in combination in the double mutants, affecting levels of different phenolics in sorghum stalks. In particular, levels of caffeic acid (CafA), an intermediate associated with the lignin biosynthetic pathway, were over three times higher in bmr6 or bmr12 Wheatland plants relative to the wild type (48). Additionally, wall-bound ferulic acid (FA), another lignin biosynthesis pathway intermediate, was $\approx 1.25$ times greater in $b m r 12$ Wheatland plants than in wild-type plants. These results show that there are differences in accumulations of phenolics in stalks of bmr plants compared with near-isogenic wild-type plants. In the present study, extent of lesion development in fungal-inoculated bmrl2 peduncles appeared to be affected by plant response (Fig. 3C; Table 3). Discoloration resulting from inoculation of sorghum peduncles, the length of which was defined as "lesion length" in the present study, are due, at least in part, to plant pigments induced during fungal infection $(10,33)$. In this study, extent of discoloration was significantly reduced in Redlan bmrl2 following inoculation by either $F$. thapsinum or F. verticillioides. However, results in Table 3 suggest that extent of fungal growth may be slightly greater, but significantly so, beyond the region of discoloration in bmrl2 plants versus wild-type plants, suggesting that bmr12 may interact with one or more factor such that the plant responds in a way which results in reduced pigmentation. Because of this interesting observation, we are conducting more extensive experiments to determine whether differential responses of $b m r$ genotypes to pathogens are a direct or indirect result of altered accumulation of pigments and other phenolic compounds.

Previous studies have shown that Bmr6 and Bmrl2 are highly homologous to their counterparts in other plants, indicating evolutionary conservation $(5,56)$. Indeed, Bmr6 was shown to be more similar to its $C A D$ gene orthologs in monocots (rice, maize, and sugarcane), dicots (Arabidopsis, tobacco, aspen, and eucalyptus), pine, and the lycophyte Salaginella than to other sorghum $C A D$ genes (57). Although fewer COMT genes were available for com- 
parison, the trend is the same; Bmrl2 was more similar to lignin biosynthesis COMT genes in pine, maize, alfalfa, and Arabidopsis than to other COMT genes in sorghum (unpublished). These results suggest that Bmr6 and Bmrl2 evolved primarily for lignin biosynthesis rather than for another function such as plant defense.

In spite of this, it is clear that lignin and related compounds could contribute to resistance. However, what about results from this and previous studies that suggest the contrary? Lignin biosynthesis is part of the phenylpropanoid pathway, which begins with the conversion of phenylalanine to cinnamic acid (CinA) by the enzyme phenylalanine ammonia lyase (PAL) (Fig. 4). CinA is a precursor of lignin monomers, salicylic acid (SA), and some phytoalexins, including 3-deoxyanthocyanidins produced by sorghum $(24,33,37)$ (Fig. 4). FA, a derivative of feruloyl CoA or coniferaldehyde, and 3-deoxyanthocyanidin phytoalexins in sorghum grain are associated with resistance to fungi (Fig. 4) $(3,61)$. During Monilinia fructicola infection of stone fruit, CafA, derived from caffeoyl CoA, reduced appressorium formation and inhibited production of the pathogenicity factors cutinase and endopolygalacturonidase (28). Because the pathways downstream of CinA are interconnected (Fig. 4), a mutation that affects one step in this phenylpropanoid metabolic network (e.g., lignin biosynthesis) could shift intermediates toward other branches, resulting in elevated levels of secondary metabolites such as aromatic phytoalexins or SA. Arabidopsis mutants impaired in COMT1, the gene orthologous to sorghum bmr12, were more susceptible to two necrotophic fungi, one biotrophic fungus, and two bacterial pathogens (52). However, they were not more susceptible to the pathogenic nematode Meloidogyne incognita, even though infection of wild-type plants induced expression of COMT1, and they were more resistant to the biotrophic oomycete Hyaloperonospora arabidopsis, as measured by a significant decrease in asexual sporulation, unstable haustoria, and an increase in oospores (sexual spores). In mutant plants, it was shown that 5-hydroxyferuloyl malate (OH-FM) accumulated to 150 to $200 \mathrm{nmol} \mathrm{g}^{-1}$ fresh tissue whereas it was undetectable in wild-type plants (52). OH-FM stimulated oospore formation in vitro in the nonobligate pathogenic oomycete Phytophthora cactorum. These results suggest that resistance in comt1 plants may be due to accumulation of $\mathrm{OH}-\mathrm{FM}$, and perhaps other metabolites, that significantly affect $H$. arabidopsis but not other pathogens. Thus, we propose that increases in antagonistic metabolites or constitutive expression of defense responses in sorghum $b m r l 2$ grain may have resulted in inhibition of colonization by FIESC no. 1319 that may have been particularly sensitive to these factors.

To summarize, this study showed that $b m r$ results in reduced colonization of sorghum grain by members of the G. fujikuroi

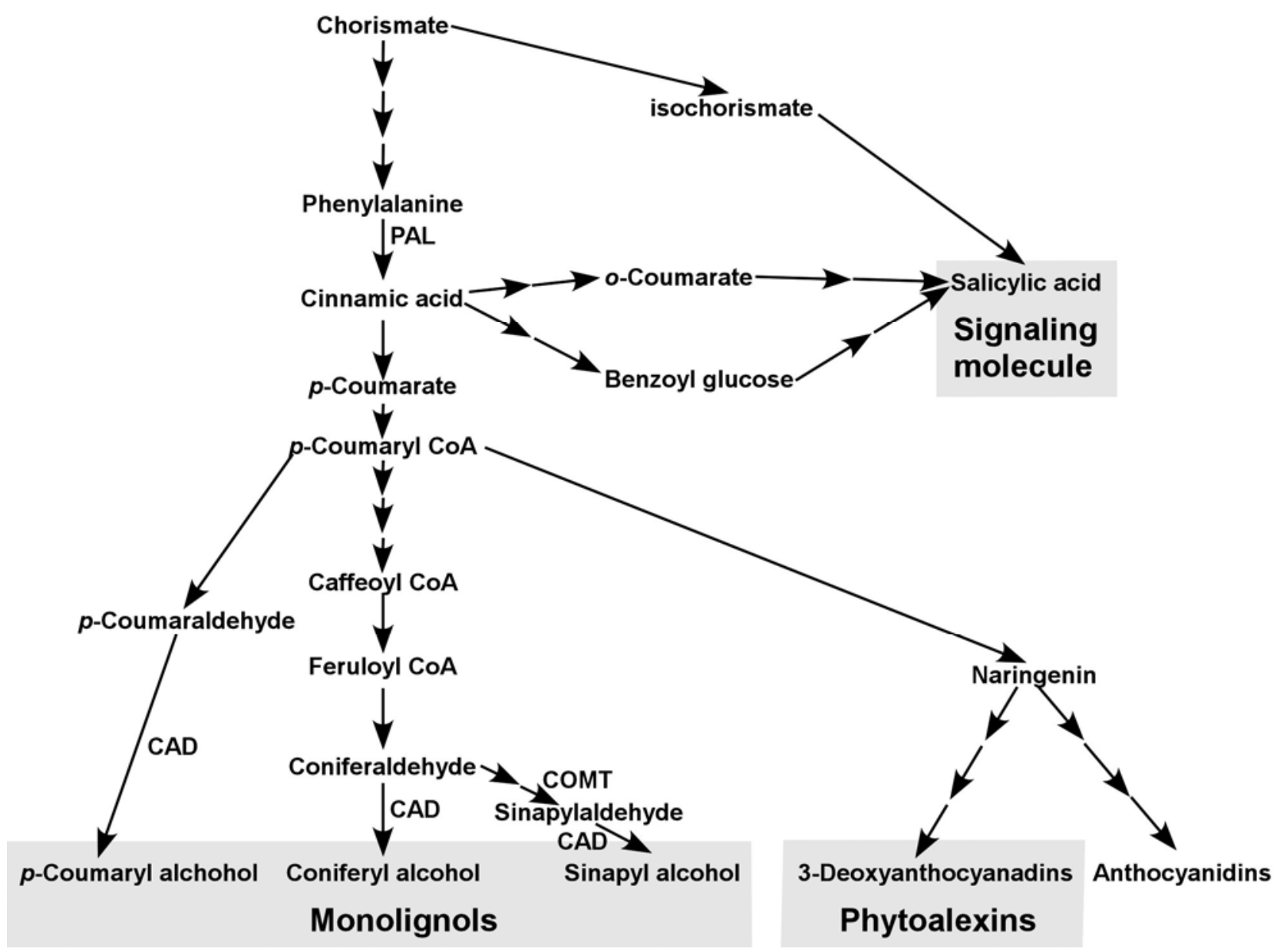

Fig. 4. Proposed pathway for synthesis of monolignols incorporated into lignin, and interactions of precursors and intermediates with the salicylic acid (SA) and 3deoxyanthocyanidin and anthocyanidin pathways. The phenylpropanoid pathway begins with the conversion of phenylalanine to cinnamic acid (CinA) by the enzyme phenylalanine ammonia lyase (PAL). $p$-Coumarate is converted to $p$-coumaryl CoA, which has three potential routes; it can be converted to $p$-coumaryl alcohol, caffeoyl CoA, or naringenin. Caffeoyl CoA is converted to coniferaldehyde, which can be converted to coniferyl alcohols or to sinapylaldehyde (21). The enzyme encoded by brown midrib (bmr)6, cinnamyl alcohol dehydrogenase (CAD), catalyzes the final step for all three monolignols. The enzyme encoded for by bmr12, caffeic acid- $O$-methyl transferase (COMT), is required for the formation of sinapyl alcohol. CinA also can be converted to SA via $o$-coumarate or benzoyl glucose, while the phenylalanine precursor, chorismate, can be converted to SA by way of isochorismate (33). Naringenin, after several steps, can be converted to 3-deoxyanthocyanidin phytoalexins and anthocyanidin pigments (30). Because bmr12 and bmr6 impaired the last two steps of monolignol biosynthesis, we propose that additional aromatic intermediates would be available for SA or aromatic phytoalexins synthesis, thus affecting response to pathogens. 
species complex, across genetic backgrounds. Also, evidence is provided that a genotype of FIESC is restricted from colonizing bmrl2 plants. Lesion lengths following inoculation of sorghum peduncles with $F$. verticillioides or A. alternata, as well as $F$. thapsinum (20), were reduced on $b m r$ plants compared with nearisogenic wild-type plants.

\section{ACKNOWLEDGMENTS}

Mention of trade names or commercial products in this article is solely for the purpose of providing specific information and does not imply recommendation or endorsement by the United States Department of Agriculture. We thank J. Toy for overseeing field operations; P. O'Neill for conducting statistical analyses; P. O’Neill and T. Gries for overseeing laboratory and greenhouse operations; D. Geiser, R. French, and K. Plummer for valuable discussions; and M. Ebeling, A. Bednar, and M. Pakes for technical assistance.

\section{LITERATURE CITED}

1. Amigot, S. L., Fulgueria C. L., Bottai, H., and Basílico, J. C. 2006. New parameters to evaluate forage quality. Postharvest Biol. Technol. 41:215224.

2. Andrews, S., and Pitt, J. I. 1986. Selective medium for isolation of Fusarium species and Dematiaceous Hyphomycetes from cereals. Appl. Environ. Microbiol. 51:1235-1238.

3. Awika, J. M., Rooney, L. W., and Waniska, R. D. 2004. Properties of 3deoxyanthocyanins from sorghum. J. Agric. Food Chem. 52:4388-4394.

4. Benson, D. A., Karsch-Mizrachi, I., Lipman, D. J., Ostell, J., and Wheeler, D. L. 2007. GenBank. Nucleic Acids Res. 36:D25-D30.

5. Bout, S., and Vermerris, W. 2003. A candidate-gene approach to clone the sorghum Brown midrib gene encoding caffeic acid $O$-methyltransferase. Mol. Gen. Genomics 269:205-214.

6. Bramel-Cox, P. J., and Claflin, L. E. 1989. Selection for resistance to Macrophomina phaseolina and Fusarium moniliforme in sorghum. Crop Sci. 29:1468-1472.

7. Campbell, M. M., and Sederoff, R. R. 1996. Variation in lignin content and composition. Plant Physiol. 110:3-13.

8. Carver, T. L. W., Zeyen, R. J., Robbins, M. P., Vance, C. P., and Boyles, D. A. 1994. Suppression of host cinnamyl alcohol dehydrogenase and phenylalanine ammonia lyase increases oat epidermal cell susceptibility to powdery mildew penetration. Physiol. Mol. Plant Pathol. 44:243-259.

9. Chisholm, S. T., Coaker, G., Day, B., and Staskawicz, B. J. 2006. Hostmicrobe interactions: shaping the evolution of the plant immune response. Cell 124:803-814.

10. Chopra, S., Gevens, A., Svabek, C., Wood, K. V., Peterson, T., and Nicholson, R. L. 2002. Excision of the Candystripe1 transposon from a hyper-mutable $Y 1$-cs allele shows that the sorghum $Y 1$ gene controls the biosynthesis of both 3-deoxyanthocyanidin phytoalexins and phlobaphene pigments. Physiol. Mol. Plant Pathol. 60:321-330.

11. Clarke, C. H., 1971. The mutagenic specificities of pentachloronitrobenzene and captan, two environmental mutagens. Mutat. Res. 11:247-248.

12. Cohen, E., Gamliel, A., and Katan, J. 1986. Glutathione and glutathioneS-transferase in fungi: effect of pentachloronitrobenzene and 1-chloro2,4-dinitrobenzene; purification and characterization of the transferase from Fusarium. Pestic. Biochem. Physiol. 26:1-9.

13. da Silva, J. B., Pozzi, C. R., Mallozzi, Ortega, E. M., and Corrêa, B. 2000. Mycoflora and occurrence of aflatoxin $\mathrm{B}_{1}$ and fumonisin $\mathrm{B}_{1}$ during storage of Brazilian sorghum. J. Agric. Food Chem. 48:4352-4356.

14. Dejene, M, Yuen, J., and Sigald, R. 2004. The impact of storage methods on storage environment and sorghum grain quality. Seed Sci. Technol. 32:511-529.

15. Delgado, N. J., Casler, M. D., Grau, C. R., and Jung, H. G. 2002. Reactions to smooth bromegrass clones with divergent lignin or etherified ferulic acid concentration to three fungal pathogens. Crop Sci. 42:18241831.

16. Dingkuhn, M., Singh, B. B., Clerget, B., Chatereau, J., and Sultan, B. 2006. Past, present and future criteria to breed crops for water-limited environments in West Africa. Agric. Water Manage. 80:241-261.

17. Duschnicky, L. G., Ballance, G. M., Sumner, M. J., and MacGregor, A. W. 1998. The role of lignification as a resistance mechanism in wheat to a toxin-producing isolate of Pyrenophora tritici-repentis. Can. J. Plant Pathol. 20:35-47.

18. Franke, R., Hemm, M. R., Denault, J. W., Ruegger, M. O., Humphreys, J. M., and Chapple, C. 2002. Changes in secondary metabolism and deposition of an unusual lignin in the ref8 mutant of Arabidopsis. Plant J. 30:47-59.
19. Funnell, D. L., Matthews, P. S., and VanEtten, H. D. 2001. Breeding for highly fertile isolates of Nectria haematococca MPVI that are highly virulent on pea and in planta selection for virulent recombinants. Phytopathology 91:92-101.

20. Funnell, D. L., and Pedersen, J. F. 2006. Reaction of sorghum lines genetically modified for reduced lignin content to infection by Fusarium and Alternaria spp. Plant Dis. 90:331-338.

21. Funnell, D. L., and Pedersen, J. F. 2006. Association of plant color and pericarp color with colonization of grain by members of Fusarium and Alternaria in near-isogenic sorghum lines. Plant Dis. 90:411-418.

22. Funnell-Harris, D. L., and Pedersen, J. F. 2008. Inoculation strategies to assess biological interactions between Fusarium and Alternaria species infecting sorghum. Can. J. Plant Pathol. 30:404-413.

23. Geiser, D. M., del Mar Jiménez-Gasco, M., Kang, S., Makalowska, I., Veeraraghavan, N., Ward, T. J., Zhang, N., Kuldau, G. A., and O'Donnell, K. 2004. FUSARIUM-id v. 1.0: a DNA sequence database for identifying Fusarium. Eur. J. Plant Pathol. 110:473-479.

24. Humphreys, J. M., and Chapple, C. 2002. Rewriting the lignin roadmap. Curr. Opin. Plant Biol. 5:224-229.

25. Jardine, D. J., and Leslie, J. F. 1992. Aggressiveness of Gibberella fujikuroi (Fusarium moniliforme) isolates to grain sorghum under greenhouse conditions. Plant Dis. 76:897-900.

26. Kawasaki, T., Koita, H., Nakatsubo, T., Hasegawa, K., Wakabayashi, K., Takahashi, H., Umemura, K., Umezawa, T., and Shimamoto, K. 2006. Cinnamoyl-CoA reductase, a key enzyme in lignin biosynthesis, is an effector of small GTPase Rac in defense signaling in rice. Proc. Natl. Acad. Sci. USA 103:230-235.

27. Kosiak, E. B., Holst-Jensen, A., Rundberget, T., Gonzalez Jaen, M. T., and Torp, M. 2005. Morphological, chemical and molecular differentiation of Fusarium equiseti isolated from Norwegian cereals. Int. J. Food Microbiol. 99:195-206.

28. Lee, M.-H., and Bostock, R. M. 2007. Fruit exocarp phenols in relation to quiescence and development of Monilinia fructicola infections in Prunus spp.: A role for cellular redox? Phytopathology 97:269-277.

29. Lee, S. B., and Taylor, J. W. 1990. Isolation of DNA from fungal mycelia and single spores. Pages 282-287 in: PCR Protocols: A Guide to Methods and Applications. M. A. Innis, D. H. Gelfand, J. J. Sninsky, and T. J. White, eds. Academic Press, Inc., San Diego, CA.

30. Leslie, J. F., Pearson, A. S., Nelson, P. E., and Tousson, T. A. 1990. Fusarium spp. from corn, sorghum, and soybean fields in the central and eastern United States. Phytopathology 80:343-350.

31. Leslie, J. F., Zeller, K. A., Lamprecht, S. C., Rheeder, J. P., and Marasas, W. F. O. 2005. Toxicity, pathogenicity, and genetic differentiation of five species of Fusarium from sorghum and millet. Phytopathology 95:275-283.

32. Li, X., Weng, J.-K., and Chapple, C. 2008. Improvement of biomass through lignin modification. Plant J. 54:569-581.

33. Lo, S.-C. C., and Nicholson, R. L. 1998. Reduction of light-induced anthocyanin accumulation in inoculated mesocotyls: Implications for a compensatory role in the defense response. Plant Physiol. 116:979-989.

34. Manthey, F. A., Wolf-Hall, C. E., Yalla, S., Vijayakumar, C., and Carlson, D. 2004. Microbial loads, mycotoxins, and quality of durum wheat from the 2001 harvest of the Northern plains region of the United States. J. Food Prot. 67:772-780.

35. McWhorter, C. G., Ouzts, C., and Paul, R. N. (1993) Micromorphology of Johnsongrass (Sorghum halapense) leaves. Weed Sci. 41:583-589.

36. Menkir, A., Ejeta, G., Butler, L. G., Melakeberhan, A., and Warren, H. L. 1996. Fungal invasion of kernels and grain mold damage assessment in diverse sorghum germ plasm. Plant Dis. 80:1399-1402.

37. Metraux, J.-P. 2002. Recent breakthroughs in the study of salicylic acid biosynthesis. Trends Plant Sci. 7:332-334.

38. Mills, J. T. 1989. Ecology of mycotoxigenic Fusarium spp. on cereal seeds. J. Food Prot. 10:737-742.

39. Nakanishi, T., and Oku, H. 1969. Metabolism and accumulation of pentachloronitrobenzene by phytopathogenic fungi in relation to selective toxicity. Phytopathology 59:1761-1762.

40. Nash, S. M., and Snyder, W. C. 1962. Quantitative estimates by plate counts of propagules of the bean root rot Fusarium in field soils. Phytopathology 52:567-572.

41. Nelson, P. E., Plattner, R. D., Shackelford, D. D., and Desjardins, A. E. 1992. Fumonisin $B_{1}$ production by Fusarium species other than $F$. moniliforme in section Liseola and by some related species. Appl. Environ. Microbiol. 58:984-989.

42. Nelson, P. E., Tousson, T. A., and Marasas, W. F. O. 1983. Fusarium Species: An Illustrated Manual for Identification. The Pennsylvania State University Press, University Park.

43. Nicholson, R. L., and Hammerschimdt, R. 1992. Phenolic compounds and their role in disease resistance. Annu. Rev. Phytopathol. 30:369-389.

44. O’Donnell, K., Cigelnik, E., and Nirenberg, H. I. 1998. Molecular systematic and phylogeography of the Gibberella fujikuroi species complex. Mycologia 90:465-493. 
45. O’Donnell, K., Sarver, B. A. J., Brandt, M., Chang, D. C., Noble-Wang, J., Park, B. J., Sutton, D. A., Benjamin, L., Lindsley, M., Padhye, A., Geiser, D. M., and Ward, T. J. 2007. Phylogenetic diversity and microsphere array-based genotyping of human pathogenic Fusaria, including isolates from the multistate contact lens-associated U.S. keratitis outbreads of 2005 and 2006. J. Clin. Microbiol. 45:2235-2248.

46. Oliver, A. L., Pedersen, J. F., Grant, R. J., Klopfenstein, T. J., and Jose, H. D. 2005. Comparative effects of the sorghum bmr-6 and $b m r-12$ genes: II. Grain yield, stover yield, and stover quality in grain sorghum. Crop Sci. 45:2240-2245.

47. Onyike, N. B. N., and Nelson, P. E. 1992. Fusarium species associated with sorghum grain from Nigeria, Lesotho, and Zimbabwe. Mycologia 84:452-458.

48. Palmer, N. A., Sattler, S. E., Saathoff, A. J., Funnell, D., Pedersen, J. F., and Sarath, G. 2008. Genetic background impacts soluble and cell wallbound aromatics in brown midrib mutants of sorghum. Planta 229:115127.

49. Pedersen, J. F., Funnell, D. L., Toy, J. J., Oliver, A. L., and Grant, R. J. 2006. Registration of twelve grain sorghum genetic stocks near-isogenic for the brown midrib genes $b m r-6$ and $b m r-12$. Crop Sci. 46:491-492.

50. Pedersen, J. F., Toy, J. J., Funnell, D. L., Sattler, S. E., Oliver, A. L., and Grant, R. A. 2008. Registration of BN611, AN612, BN612, and RN613 sorghum genetic stocks with stacked $b m r-6$ and $b m r-12$ genes. J. Plant Regist. 2:258-262.

51. Peltier, A. J., Hatfield, R. D., and Grau, C. R. 2009. Soybean stem lignin concentration relates to resistance to Sclerotinia sclerotiorum. Plant Dis. 93:149-154.

52. Quentin, M., Allasia, V., Pegard, A., Allais, F., Ducrot, P.-H., Favery, B., Levis, C., Martinet, S., Masur, C., Ponchet, M., Roby, D., Schlaich, N. L., Jouanin, L., and Ketter, H. 2009. Imbalanced lignin biosynthesis promotes the sexual reproduction of homothallic oomycete pathogens. PLoS Pathog. 5:e1000264.

53. Saballos, A., Ejeta, G., Sanchez, E., Kang, C. H., and Vermerris, W. 2009. A genomewide analysis of the cinnamyl alcohol dehydrogenase family in sorghum [Sorghum bicolor (L.) Moench] identifies SbCAD2 as the Brown midrib6 gene. Genetics 181:783-795.

54. Sarath, G., Mitchell, R. B., Sattler, S. E., Funnell, D., Pedersen, J. F., Graybosch, R. A., and Vogel, K. P. 2008. Opportunities and roadblocks in utilizing forages and small grains for liquid fuels. J. Ind. Microbiol. Biotechnol. 35:343-354.

55. Sashidhar, R. B., Ramakrishna, Y., and Bhat, R. V. 1992. Moulds and mycotoxins in sorghum stored in traditional containers in India. J. Stored Prod. Res. 28:257-260.

56. Sattler, S. E., Funnell-Harris, D. L., and Pedersen, J. F. 2010. Efficacy of singular and stacked brown midrib 6 and 12 in the modification of lignocelluloses and grain chemistry. J. Agric. Food Chem. 58:3611-3618.

57. Sattler, S. E., Saathoff, A. J., Haas, E. J., Palmer, N. A., Funnell-Harris, D. L., Sarath, G., and Pedersen, J. F. 2009. A nonsense mutation in a cinnamyl alcohol dehydrogenase gene is responsible for the sorghum brown midrib 6 phenotype. Plant Physiol. 150:584-595.

58. Tarekegn, G., McLaren, N. W., and Swart, W. J. 2006. Effects of weather variables on grain mould of sorghum in South Africa. Plant Pathol. 55:238-245.

59. Tesso, T. T., Claflin, L. E., and Tuinstra, M. R. 2004. Estimation of combining ability for resistance to Fusarium stalk rot in grain sorghum. Crop Sci. 44:1195-1199.

60. Voss, K. A., Smith, G. W., and Haschek, W. M. 2007. Fumonisins: toxicokinetics, mechanism of action and toxicity. Anim. Feed Sci. Technol. 137:299-325

61. Waniska, R. D., Poe, J. H., and Bandyopadhyay, R. 1989. Effects of growth conditions on grain molding and phenols in sorghum caryopsis. J. Cereal Sci. 10:217-225.

62. Wuyts, N., Lognay, G., Verscheure, M., Marlier, M., De Waele, D., and Swennen, R. 2007. Potential physical and chemical barriers to infection by the burrowing nematode Radopholus similis in roots of susceptible and resistant banana (Musa spp.) Plant Pathol. 56:878-890.

63. Zaccardelli, M., Balmas, V., Altomare, C., Corazza, L., and Scotti, C. 2006. Characterization of Italian isolates of Fusarium semitectum from alfalfa (Medicago sativa L.) by AFLP analysis, morphology, pathogenicity and toxin production. J. Phytopathol. 154:454-460. 